\title{
Introduction to Fractal Antenna
}

\author{
Syed Mohd. Adnan ${ }^{1}$, Pallavi Asthana ${ }^{2}$, O.P. Singh $^{3}$, Sumita Mishra ${ }^{4}$, Mohd Maroof Siddiqui ${ }^{5}$ \\ Department of Electrical \& Electronics Engineering, Amity University, Uttar Pradesh ${ }^{1,2,3,4}$ \\ Integral University, Lucknow ${ }^{5}$
}

\begin{abstract}
In this article, the most recent improvements in the field of fractal antenna designing are theoretically and additionally basically checked on and its future extension is talked about. Fractal design is a geometric example that is rehashed at each design and in this way can't be spoken to by exemplary geometry. The method of considering the fractal geometry in designing various attributes related to the performance of the antenna becomes advantageous when it improves the some parameters such as low VSWR, high radiation efficiency, high gain, wide bandwidth and reduced size etc. Fractal has exceptional property that it can make duplicates of itself at various scales. Fractal antenna basically centered around two fields: one is the utilizations of fractal antenna and second is the design and investigation of fractal antenna. As of late there has been a lot of enthusiasm for the design of antenna for remote correspondence on account of continuously growing scope of remote telecom benefits and related applications for voice and information transmission. The motivation behind this paper is to audit various inventive configuration ideas of Fractal antenna for remote applications.
\end{abstract}

Keywords: Fractal, Fractal Antenna, VSWR.

\section{INTRODUCTION}

The development of reception apparatuses with fractal geometries has given a response to two of the principle confinements expressed by Werner (1999) of the established radio wires, which are the single band execution and the reliance in the middle of size and working recurrence. The expression "fractal", implies broken or sporadic sections. It was initially instituted by Mandelbrot (1983) to portray a group of complex shapes that have a natural self-comparability or self-proclivity in their geometrical structure. Jaggard (1990) characterized fractal electrodynamics as a zone in which fractal geometry was joined with electromagnetic hypothesis with the end goal of researching another class of radiation, proliferation, and dissipating issues. A standout amongst the most encouraging regions of fractal electrodynamics examination is in its application to reception apparatus hypothesis and configuration. There are assortments of methodologies that have been created throughout the years, which can be used to accomplish one or a greater amount of these outline targets. The advancement of fractal geometry came generally from an inside and out investigation of the examples of nature. With the development of remote correspondence frameworks and their expanding significance, wideband and low profile receiving antennas are in extraordinary interest for both business and military applications.

Multiband and wideband radio wires are alluring in individual correspondence frame works; little satellite correspondence terminals, and different remote applications. Some of these applications additionally require a radio wire to be implanted into the airframe structure. Customarily, a wideband receiving antenna in the low recurrence remote groups must be accomplished with vigorously stacked wire radio wires, which more often than not implies that diverse reception apparatuses are required for various recurrence groups. Late advance in the investigation of fractal radio wires proposes some

alluring answers for utilizing a solitary little reception apparatus working in a few recurrence groups. The selfcomparable properties of specific fractals result in a multiband conduct of the radio antennas while; the exceedingly convoluted state of these fractals makes conceivable the diminishment in size, and subsequently in mass and volume, of specific reception apparatuses as examined by Puente et al. (1998)

The expression "fractal" was found by the French mathematician B.B. Mandelbrot in 1970. A "fractal" is a geometrical shape that can be part into parts, each of which is a diminished size duplicate of the entire vastly. Fractals are a class of shapes which have not trademark size. Every fractal is made out of numerous cycles of a solitary shape. The emphasis can proceed interminably, in this manner framing a shape inside of a limited limit however of boundless length or region. [3] The utilization of fractal geometries are utilized as a part of numerous ranges of science and designing; one of which is receiving antennas. Radio antennas utilize some of these geometries for different correspondence applications. The utilization of fractal geometries has been appeared to enhance a few reception apparatus elements to shifting degrees. For decreasing the extent of radio wire, fractal geometries have been presented. Present day telecom framework requires reception apparatuses with more extensive transmission capacity and littler measurements than ordinarily possible. This has started radio antenna research in different directions, one of which is by utilizing fractal formed antenna components. In present world situation remote correspondence gets to be one of the quick going advances for everybody and they inclines towards this innovation in view of the pace regarding information rate. There are some other application like satellite correspondence in this time weight is a standout amongst the most critical imperative, GPS ,Advanced military framework, requires reception apparatuses of required 
execution, expansive increase, more extensive data At transmission, multiband, minimal effort and traditionally littler configuration measurements. Analysts have been doing their exploration to accomplish necessities towards this innovation. Among them Dr. Nathan Cohen is the organizer and innovation visionary behind Fractal Antenna Systems In 1988, Dr. Cohen fabricated the world's first fractal radio wire and, he is dealing with this innovation. He established Fractal Antenna in 1995 to spread his exploration in business and also military applications. He has one of the world's celebrated inventive creators in the field receiving antenna planning radio wire with fractal component reception apparatuses ended up being much more decreased size, solid and more valuable than customary reception apparatus outlines. Fractal reception apparatus can address the issue receiving antenna necessities of advanced correspondence, exceptionally contract segment, conservative size, being anything but difficult to make and low cost.

\section{FRACTAL SHAPED ANTENNA ELEMENTS}

The way of fractal geometries has gotten the consideration of radio wire fashioners, fundamentally as a past-time. However with the extending of comprehension of reception apparatuses utilizing them a few geometrical and radio wire highlights have been between connected. This has prompted the development of another class of reception apparatuses, called fractal molded radio wires. Cohen has attempted the helpfulness a few fractal geometries tentatively. Koch bends, Murkowski bends, Sierpinski gasket are among them. The main fractal that will he considered is the prominent Sierpinski gasket [14].The initial few stages in the development of the Sierpinski gasket are appeared in Figure 1.1 Another prevalent fractal is known as the Koch snowflake [14]. This fractal additionally begins as a strong equilateral triangle in the plane, as showed in of Figure 1.1 number of structures taking into account absolutely deterministic or arbitrary fractal trees has likewise turned out to be amazingly helpful in growing new outline procedures for reception apparatuses and recurrence particular surfaces. A sample of a deterministic tenary (three branches) fractal tree is appeared in Figure 1.3.The Koch snowflakes and islands have been basically used to grow new outlines for scaled down circle and also Micro-strip Patch Antennas. New outlines for scaled down dipole reception apparatuses have additionally been produced in light of an assortment of Koch bends and fractal trees.
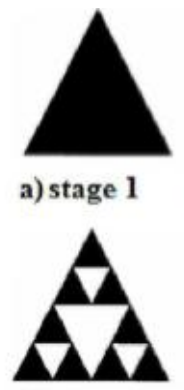

c) stage 3

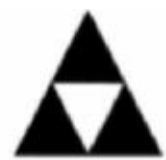

b) stage 2

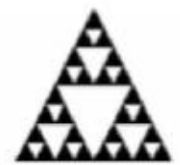

d) stage 4
Fig 1.1Stages of Sierpinski Gasket Fractal
At long last, the self-comparable structure of Sierpinski gaskets and floor coverings has been misused to create multi-band radio wire components as can be seen below in the figures.

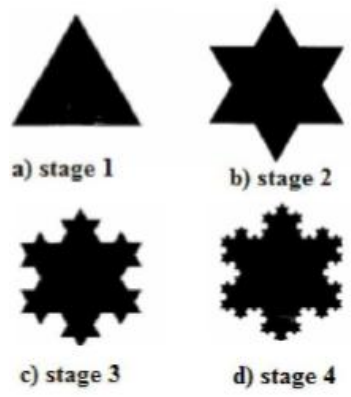

Fig 1.2 Stages of Koch Fractal

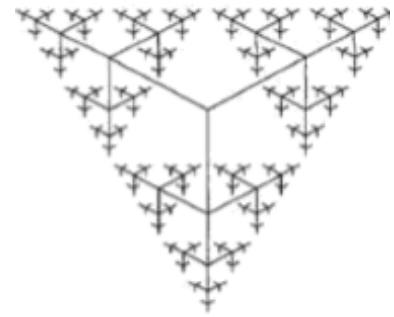

Fig 1.3 A stage of Ternary fractal tree

\section{FEATURES OF FRACTAL ANTENNAS}

As of now said the fractal radio wires utilize the fractal geometry for their outline when contrasted with established reception apparatuses which utilize Euclidean Geometry. The two essential properties of fractals give recognized components to these fractal planned reception apparatuses, these are talked about with fitting application regions below:

\section{MULTIBAND/ WIDEBAND PERFORMANCE}

Any great reception apparatus framework requires receiving antenna scaling which implies that the distinctive parameters (impedance, radiation pattern, bandwidth, gain and so on.) stay same if every one of the measurements and the wavelength are scaled by same variable. Following because of self-likeness controlled by fractals, the fractal structure give off an impression of being same autonomous of size scaling and along these lines it can be deciphered that the fractal structures can be utilized to acknowledge radio wire plans over a substantial band of frequencies [8]. The reception apparatus can be worked also at different frequencies which imply that the receiving antenna keeps the comparative radiation parameters through a few groups.

\section{COMPACT SIZE}

Another prerequisite for antenna plan in remote frameworks is the minimal size. The partial measurement and space filling property of fractal shapes permit the fractal molded antennas to use the little encompassing space successfully [9]. This likewise conquers the constraint of execution of little traditional radio wires and aides in keeping up smaller size in reception apparatus which permits its application in cell handsets. Since fractal 
receiving antennas are more reduced, they fit all the more effortlessly in the beneficiary bundle. As of now, numerous cell handsets use quarter wavelength monopoles which are basically segments of transmitting wires slice to a decided length. Albeit straightforward, they have brilliant radiation properties. Nonetheless, for frameworks working at high frequencies, for example, GSM, the length of these monopoles is regularly more than the handset itself. It would be very gainful to outline a receiving antenna in light of fractal configuration with comparable radiation properties as the quarter wavelength monopole while holding its radiation properties.

\section{RECONFIGURABILITY}

Reconfigurability in reception apparatus frameworks is a fancied element that has as of late gotten significantly consideration in creating novel and spearheading multifunctional radio wire plans. Contrasted with routine radio wires, reconfigurable receiving antennas give the capacity to progressively modify different reception apparatus parameters. The dynamic tuning of such manipulating so as to receiving antenna parameters are regularly accomplished a specific exchanging conduct. Reconfigurable receiving antennas lessen any unfavorable impacts coming about because of co-site impedance and sticking (Peroulis et al., 2005). Moreover, they have an exceptional normal for accomplishing differing qualities in operation, implying that one or numerous parameters, including working recurrence, radiation example, pick up and/or polarization can be reconfigured with a solitary receiving antenna. The utilization of reconfigurability in a joint effort with a self-comparable receiving antenna prompts an extensive change in reception apparatus execution. This is on account of not just a more extensive choice of frequencies is accomplished, additionally comparative radiation properties for all outlined recurrence groups

\section{ADVANTAGES AND DISADVANTAGES}

The various advantages of fractal antennas can be listed as:

- Smaller cross sectional area

- No impedance matching network required

- Multiple resonances

- Higher gain in some cases

Although in the early stage of their development, these antenna designs suffer from two main disadvantages. These are:

- Fabrication and design is little complicated

- Lower gain in some cases

- Mathematical limitations

Further investigations and new developments in this field may be helpful in overcoming these disadvantages.

\section{APPLICATIONS OF FRACTAL ANTENNAS}

There are numerous applications that can profit by fractal reception apparatuses. Examined underneath are a few thoughts where fractal reception apparatuses can have a genuine effect. The sudden develop in the remote correspondence territory has sprung a requirement for reduced incorporated receiving antennas. The space sparing capacities of fractals to proficiently fill a restricted measure of space make unmistakable point of interest of utilizing coordinated fractal reception apparatuses over Euclidean geometry. Cases of these sorts of use incorporate individual hand-held remote gadgets, for example, phones and different remote cell phones, for example, portable PCs on remote LANs and networkable PDAs. Fractal radio wires can likewise improve applications that incorporate multiband transmissions. This zone has numerous conceivable outcomes running from double mode telephones to gadgets coordinating correspondence and area administrations, for example, GPS, the worldwide situating satellites. Fractal receiving antennas additionally diminish the zone of a thunderous radio wire, which could bring down the radar cross-area (RCS).

\section{CONCLUSION}

Numerous varieties of fractal geometries have been fused into the configuration of reception apparatuses. Further work is required to get a comprehension of the relationship between the execution of the radio wire and the fractal measurement of the geometry that is used in its development. This requires two condemnations of activity. The principal strategy requires that numerous more samples of fractal geometries are connected to receiving antennas. The second vital game-plan is to achieve a superior comprehension of the fractal measurement of the geometries such that relationships can be drawn about this measurement and the execution of the antenna. Likewise vital is that the outline of the antenna approaches a perfect fractal however much as could be expected. A few cycles can be examined to comprehend the patterns that oversee the reception apparatus.

\section{REFERENCES}

[1] Z. Baharav Z., "Fractal Arrays Based on Iterated Function Systems (IFS)," IEEE International Symposium on Antennas and Propagation Digest, Orlando, Florida, vol. 4, pp.2686-2689, 1999.

[2] D. Baldacci and D. H. Werner, "An Efficient Recursive Procedure for Calculating the Driving Point Impedance of Linear and Planar Fractal Arrays", IEEE International Symposium on Antennas and Propagation Digest, Boston, Massachusetts ,vol. 3, pp. 620-623, 2001

3] M.F. Bamsley, Fractals Everywhere, Second Edition, Academic Press Professional, New York, 1963.

[4] C. Borja, C. Puente, and A. Median, "Iterative Network Model, to predict the Behavior of a Sierpinski Fractal Network," IEE Electronics Letters, vol. 34, pp. 1443-1445, 1998.

[5] C. Borja and J. Romen, "Multiband Sierpinski Fractal Patch Antenna', IEEE International Symposium on Antennas and Propagation Digest, Salt Lake City, Utah, vol. 3, pp.1708-1711, 2000.

[6] L. J. Chu, "Physical limitations of omni-directional antennas", J. Appl. Physics,vol. 19,pp-1163-1175, 1948.

[7] N. Cohen, "Fractal Antennas: Part I," Communications Quarterly, Summer, pp-7-22, 1995.

[8] N. Cohen, "Fractal Antennas: Part 2," Communications Quarterly, Summer, pp. 53-66, 1996.

[9] S. E. El-Khamy, M.A. Aboul-Dahab, and M. I. Elkashlan, "A Simplified Koch Multiband Fractal Array Using Windowing and Quantization Techniques", IEEE International Symposium on Antennas and Propagation Digest, Salt Lake City, Utah, vol. 3, pp. 1716-1719, 2000. 
[10] Siddiqui, Mohd Maroof. "Vision of 5G Communication." High Performance Architecture and Grid Computing. Springer Berlin Heidelberg, 2011. 252-256.

[11] Mantri, Archana, et al., eds. High Performance Architecture and Grid Computing: International Conference, HPAGC 2011, Chandigarh, India, July 19-20, 2011. Proceedings. Vol. 169. Springer, 2011.

[12] Misra, Anand Mohan, et al. "APPLICATION OF "MECHATRONICS" ALPHA I (FIRE FIGHTING ROBOT)." INTERNATIONAL JOURNAL OF ENGINEERING SCIENCE \& ADVANCED TECHNOLOGY (IJESAT)2.4(2012): 831 - 835

[13] Siddiqui, Mohd Atif, and Mohd Maroof Siddiqui. "Mathematical modelling and analysis of an Actuator for aerospace vehicle." International Journal for Science and Research in Technology (IJSART) 1.7(2015):31-34

[14] J. P. Gianvittorio and Y. Rahmat-Samii, "Fractal Elements in Array Antennas: Investigating Reduced Mutual Coupling and Tighter Packing", IEEE international Symposium on Antennas and Propagation Digest Salt Lake City, Utah, vol 3, pp.1704- 1707, 2000.

[15] C.A Balanis, Antenna Theory: Analysis and Design, New York: Wiley second ed., 1997.

[16] W.T Wang, S.X Gong, X. Wang, Y. Guan and W. Jiang, "Differential evolution algorithm and Method of Moments for the design of low RCS antennas," IEEE Antennas and Wireless Propagation Letters,vol.9, 2010, pp. 295-298.

[17] Best, S.R, and J.D., Morrow, "The effectiveness of spacefilling fractal geometry in lowering resonant frequency," IEEE Antennas and Wireless Propagation Letters, vol.1, 2002, pp. 112-115.

[18] Ankush kansal and Jaspreet Kaur, "Sierpinski Gasket Fractal Array Antenna," International Journal of Computer Science \& Communication, vol.1, no.2, July-December 2010, pp. 133-136.

[19] Kimtouche, Abed, Atrouz, and Akas, "Banwidth enhancement of monopole antenna using modified semielliptical ground plane \& slots," Microw. Opt Technol. Letter, vol.52, no.1, January 2010, pp. $54-58$.

[20] Pourahmadazar, Ghobadi and Nourinia, "Novel Modified Pythagorean Tree Fractal Monopole Antennas for UWB appplications," IEEE Antennas and Wireless Propagation Letters, vol.10, 2011.

[21] C.P Baliarda, J.Romeu, and A. Cardams, "The Koch monopole: A small fractal antennas," IEEE Trans. Antennas \& Propagation, vol.48, November 2002, pp. 1773-1781.

[22] Romen \& J.Soler, "Generalized Sierpinski Fractal Multiband antennas," IEEE Trans. Antennas \& Propagation, vol.49, no.8 August 2001, pp. 1237-1239.

[23] Sarita Verma, Aishwarya Verma, "A Review of Miniature Fractal Antenna Design for Wireless Communication," IJAREEIE, vol.3, issue 9, September 2014. 